\title{
SiO EMISSION AS A TRACER OF X-RAY DOMINATED CHEMISTRY IN THE GALACTIC CENTER
}

\author{
M. A. Amo-Baladrón ${ }^{1}$, J. Martín-Pintado ${ }^{1}$, M. R. Morris ${ }^{2}$, M. P. Muno ${ }^{3}$, And N. J. Rodríguez-Fernández ${ }^{4}$ \\ ${ }^{1}$ Centro de Astrobiología (CSIC-INTA), Ctra de Torrejón a Ajalvir, km 4, 28850 Torrejón de Ardoz, Madrid, Spain; arancha@damir.iem.csic.es, \\ jmartin.pintado@iem.cfmac.csic.es \\ 2 Department of Physics \& Astronomy, University of California, Los Angeles, CA 90095-1547, USA; morris@astro.ucla.edu \\ ${ }^{3}$ Space Radiation Laboratory, California Institute of Technology, Pasadena, CA 91104, USA; mmuno@srl.caltech.edu \\ ${ }^{4}$ Institut de Radioastronomie Millimetrique (IRAM), 300 rue de la Piscine, 38406 Saint Martin d'Heres, France; rodriguez@iram.fr \\ Received 2008 July 2; accepted 2008 December 30; published 2009 March 20
}

\begin{abstract}
We present emission maps of the Sgr A molecular cloud complex at the Galactic center (GC) in the $J=2 \rightarrow 1$ line of $\mathrm{SiO}$ observed with the IRAM $30 \mathrm{~m}$ telescope at Pico Veleta. Comparing our $\mathrm{SiO}(2-1)$ data cube with that of $\mathrm{CS}(1-0)$ emission with similar angular and velocity resolution, we find a correlation between the $\mathrm{SiO} / \mathrm{CS}$ line intensity ratio and the equivalent width of the $\mathrm{Fe} \mathrm{K} \alpha$ fluorescence line at $6.4 \mathrm{keV}$. We discuss the $\mathrm{SiO}$ abundance enhancement in terms of the two most plausible scenarios for the origin of the $6.4 \mathrm{keV} \mathrm{Fe} \mathrm{line:} \mathrm{X-ray} \mathrm{reflection}$ nebula (XRN) and low-energy cosmic rays (LECRs). Both scenarios could explain the enhancement in the $\mathrm{SiO} / \mathrm{CS}$ intensity ratio with the intensity of the $6.4 \mathrm{keV} \mathrm{Fe} \mathrm{line,} \mathrm{but} \mathrm{both} \mathrm{present} \mathrm{difficulties.} \mathrm{The} \mathrm{XRN} \mathrm{scenario} \mathrm{requires} \mathrm{a}$ population of very small grains to produce the $\mathrm{SiO}$ abundance enhancement, together with a past episode of bright X-ray emission from some source in the GC, possibly the central supermassive black hole, SgrA*, 300 yr ago. The LECR scenario needs higher gas column densities to produce the observed $6.4 \mathrm{keV} \mathrm{Fe} \mathrm{line} \mathrm{intensities} \mathrm{than} \mathrm{those}$ derived from our observations. It is possible to explain the $\mathrm{SiO}$ abundance enhancement if the LECRs originate in supernovae and their associated shocks produce the $\mathrm{SiO}$ abundance enhancement. However, the LECR scenario cannot account for the time variability of the $6.4 \mathrm{keV}$ Fe line, which can be naturally explained by the XRN scenario.
\end{abstract}

Key words: cosmic rays - Galaxy: center - ISM: clouds - ISM: molecules - radio lines: galaxies - X-rays: ISM

\section{INTRODUCTION}

Active galactic nuclei (AGNs) are in general strong emitters of X-rays arising in the accretion disks of supermassive black holes. It has been proposed that the chemical complexity of molecular clouds in galaxies having AGNs is affected by their interaction with those X-rays, producing X-ray dominated regions (XDRs; Meijerink et al. 2007). When X-ray radiation impinges on molecular clouds, the ionization degree increases, generating a specific chemistry in the XDRs (Maloney et al. 1996; Lepp \& Dalgarno 1996; Spaans \& Meijerink 2005). In addition to the chemical effects, in the presence of hard X-rays one also expects to observe the iron $\mathrm{K} \alpha$ line at $6.4 \mathrm{keV}$ (hereafter $\mathrm{Fe}^{\circ}$ line) from the XDR. This line is produced by fluorescence caused by X-rays or high energy particles ( $>7.1 \mathrm{keV})$ interacting with neutral or partially ionized iron atoms (below Fe XVII). As a result, a K-shell electron is removed from the iron atom and the filling of this inner-shell vacancy produces the fluorescence line emission at $6.4 \mathrm{keV}$ with a probability of 0.3 (Sunyaev \& Churazov 1998).

The Galactic center (GC) contains a great variety of sources of energetic activity including a massive black hole, supernova remnants (SNRs), several massive stellar clusters, strong magnetic fields, and a strong background of X-ray emission due to a combination of a multitemperature plasma and a large population of X-ray binary stars, both of which are distributed throughout the whole region (Muno et al. 2003; see Morris \& Serabyn 1996 and Mezger et al. 1996 for reviews of the region). The GC also shows strong emission of the $\mathrm{Fe}^{\circ}$ line (Koyama et al. 1996), but its origin is still a matter of discussion (see, e.g., Koyama et al. 2008; Yusef-Zadeh et al. 2007 and references therein). The Advanced Satellite for Cosmology and Astrophysics (ASCA) map of the $\mathrm{Fe}^{\circ}$ line showed that this emission was mainly concentrated in the Sgr B and Sgr A molecular cloud complexes (Koyama et al. 1996). Large-scale mapping of the 200 central parsecs of our Galaxy in the $J=1 \rightarrow 0$ line of $\mathrm{SiO}$, with angular resolution similar to that of the ASCA map $\left(2^{\prime}\right)$, has shown a correlation between the spatial distribution of $\mathrm{SiO}$ emission and the intensity of the $\mathrm{Fe}^{\circ}$ line (Martín-Pintado et al. 2000).

It is well established that the $\mathrm{SiO}$ abundance is strongly enhanced by the sputtering of grains and grain mantles by shocks generated by energetic phenomena (Martín-Pintado et al. 1992). However, Martín-Pintado et al. (2000) have also found that within the Sgr B2 complex the intensity ratio between the $\mathrm{SiO}$ $J=1 \rightarrow 0$ and the CS $J=2 \rightarrow 1$ emission increases by more than a factor of 20 in the region with strong $\mathrm{Fe}^{\circ}$ line emission. They claimed that an important fraction of the $\mathrm{SiO}$ emission from the molecular clouds in that region is due to the enhancement of the $\mathrm{SiO}$ abundance in the XDRs traced by the $\mathrm{Fe}^{\circ}$ line emission.

With the advent of the new generation of X-ray telescopes, Chandra and XMM-Newton, new high angular resolution imaging of the $\mathrm{Fe}^{\circ}$ line has became available (Koyama et al. 2003; Predehl et al. 2003; Park et al. 2004). Mapping of the CS(1-0) line, carried out by Tsuboi et al. (1999), has shown that the morphology of the molecular emission from the G $0.11-0.11$ molecular cloud in the Sgr A complex is very similar to that of the $\mathrm{Fe}^{\circ}$ line (Yusef-Zadeh et al. 2002; Koyama et al. 2003), suggesting a clear association between the dense molecular gas and the X-ray emission. However, the $\mathrm{Fe}^{\circ}$ line emission in the Sgr A complex is not restricted only to the G $0.11-0.11$ cloud, but is much more extended, showing also very strong emission toward the region of the Arched Thermal Filaments and the Arches Cluster.

In this paper, we present a mapping of the Sgr A molecular cloud complex in $\mathrm{SiO} J=2 \rightarrow 1$ emission with a spatial resolution $\sim 40^{\prime \prime}$. We report that the $\mathrm{SiO}(2-1) / \mathrm{CS}(1-0)$ intensity ratio systematically increases as the $\mathrm{Fe}^{\circ}$ line intensity increases, 


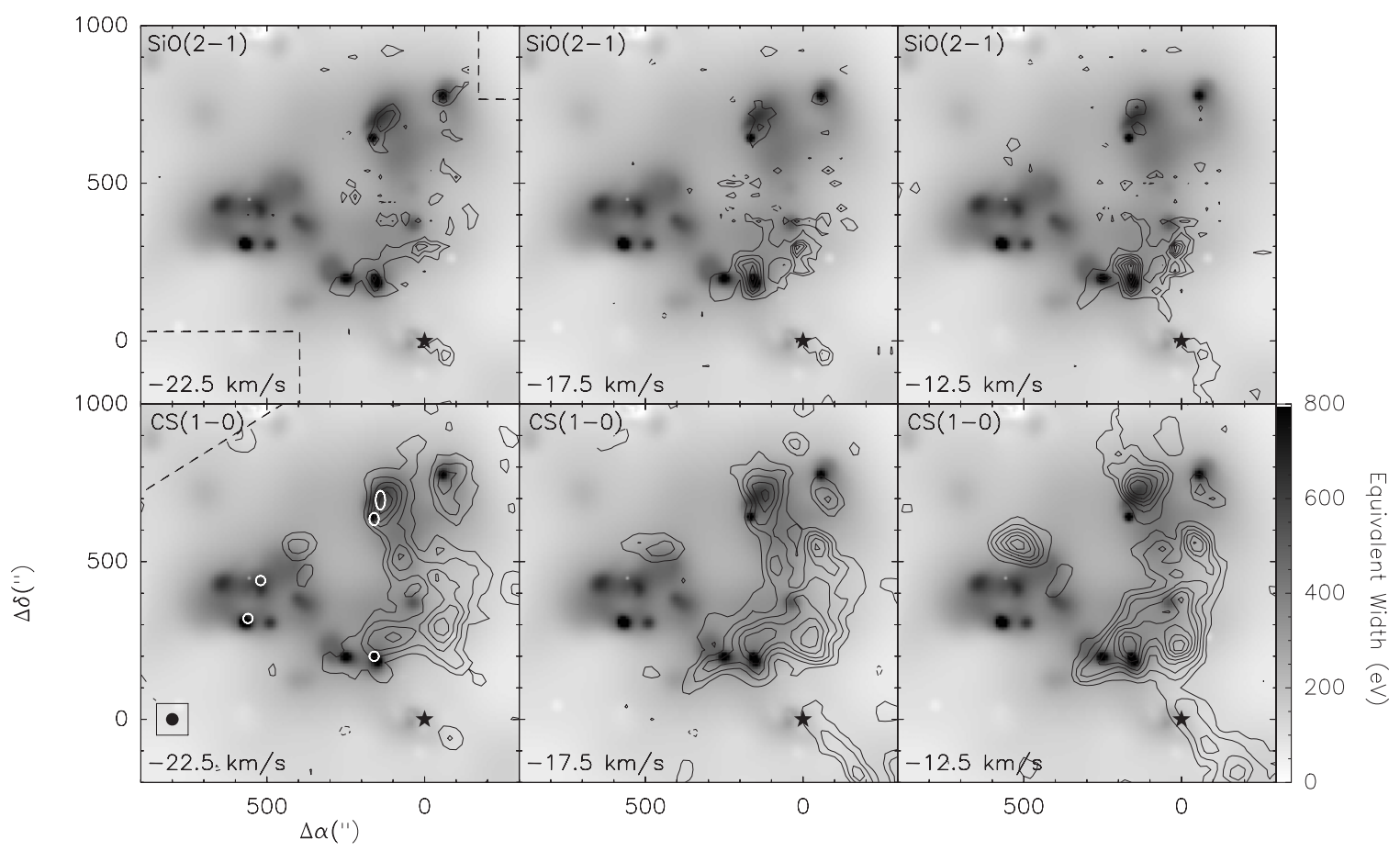

Figure 1. Spatial distribution of the $\mathrm{SiO}(2-1)$ (upper panels) and $\mathrm{CS}(1-0)$ (lower panels) line emission superimposed on the image of the Fe ${ }^{\circ}$ line (Sgr A XDR) shown in gray scale for the negative velocity channel maps. Contour levels of the $\mathrm{SiO}(2-1)$ emission (in $T_{A}^{*}$ scale) are from $0.12 \mathrm{~K} \mathrm{~km} \mathrm{~s}^{-1}(3 \sigma)$ in steps of $0.10 \mathrm{~K} \mathrm{~km} \mathrm{~s}{ }^{-1}$. Contour levels of the CS(1-0) emission are from $0.30 \mathrm{~K} \mathrm{~km} \mathrm{~s}^{-1}(3 \sigma)$ in steps of $0.20 \mathrm{~K} \mathrm{~km} \mathrm{~s}^{-1}$. The central velocities of the line emission maps are shown in the bottom left corner of each panel. The velocity width per channel is $5 \mathrm{~km} \mathrm{~s}^{-1}$. The gray scale of the $\mathrm{Fe}^{\circ}$ image, shown in the wedge at right, corresponds to the EW of this line. This scale is saturated to emphasize the weaker features, and to show more clearly the intensity maxima of the $\mathrm{Fe}^{\circ}$ line. The filled star marks the position of Sgr A*, which is the origin for the offset coordinates in arcseconds. The dashed lines show the boundaries of the molecular maps, and the beam size is drawn inside a square in the bottom left panel. In the this panel, we also show the location of the regions (white ellipses) and points (white circles with a diameter equal to the beam size at the $\mathrm{SiO}(2-1)$ frequency) corresponding to the selected spectra of Figure 5.

suggesting that the $\mathrm{SiO}$ abundance is enhanced as a result of the same energetic mechanisms that lead to emission in the $\mathrm{Fe}^{\circ}$ line.

\section{OBSERVATIONS AND RESULTS}

The mapping of the $J=2 \rightarrow 1$ line of $\mathrm{SiO}$ was carried out with the IRAM-30m radiotelescope at Pico Veleta (Spain). The data were obtained in several observing periods, with the last one in 2006. The half-power beamwidth (HPBW) of the telescope was $29^{\prime \prime}$ at the rest frequency of the transition $(86846.96 \mathrm{MHz})$. The receivers were tuned to a single sideband with image rejections $>10 \mathrm{~dB}$. The system temperatures ranged between 160 and $560 \mathrm{~K}$. We used filterbanks as spectrometers, providing a spectral resolution of $3.4 \mathrm{~km} \mathrm{~s}^{-1}$. Within the same bandwidth, we simultaneously observed the $J=1 \rightarrow 0$ line of $\mathrm{H}^{13} \mathrm{CO}^{+}$at 86754.33 MHz. Spectra were calibrated using the standard dual load system. We used the antenna temperature scale $\left(T_{A}^{*}\right)$ for the line intensities because the emission is rather extended and completely fills the main beam.

The bulk of the data were observed in raster mode with the reference position located far away from the Galactic plane. The positions of the raster grid were separated by $40^{\prime \prime}$, although in some regions this separation was $20^{\prime \prime}$. The most recent images of selected regions (including the Thermal Filaments) were obtained using the On-The-Fly (OTF) mode and they were fully sampled. All the data were finally combined and regridded using a Gaussian kernel with a HPBW of $40^{\prime \prime}$. The final channel maps $(49 \times 49 \mathrm{pc}$ at the GC distance of $8.5 \mathrm{kpc})$ cover the main features in the Sgr A complex, including the whole molecular region with $\mathrm{Fe}^{\circ}$ line emission observed by Chandra (YusefZadeh et al. 2007).

Additionally, we also present observations of the $J=3 \rightarrow$ 2 line of $\mathrm{SiO}$ at selected positions (marked with white circles and ellipses in Figure 1). The HPBW of the telescope at the rest frequency of this transition $(130268.61 \mathrm{MHz})$ was $19^{\prime \prime}$, and the system temperatures ranged between 267 and $595 \mathrm{~K}$.

The upper panels in Figures 1 and 2 show the $\mathrm{SiO} J=2 \rightarrow 1$ emission as contour levels for six different radial velocities (see Section 3 for a discussion of the selection) superimposed on the image of the equivalent width (EW) of the $\mathrm{Fe}^{\circ}$ line emission from the X-ray satellite Chandra (Yusef-Zadeh et al. 2007). In the lower panels we show the corresponding CS $J=1 \rightarrow 0$ line images (in contours) from Tsuboi et al. (1999) also superimposed on the EW map of the $\mathrm{Fe}^{\circ}$ line. As already discussed by Martín-Pintado et al. (2000) for the Sgr $\mathrm{B}$ complex, the $\mathrm{Fe}^{\circ}$ line is only associated with a subset of the molecular clouds located along any given line of sight, which can be identified by their radial velocities. Unfortunately, the $\mathrm{X}$-ray spectroscopy lacks the energy resolution necessary to compare directly the $\mathrm{Fe}^{\circ}$ and the molecular line emission for different radial velocities.

\section{MOLECULAR CLOUDS ASSOCIATED WITH THE Fe ${ }^{\circ}$ LINE}

To identify which molecular radial velocities are associated with the $\mathrm{Fe}^{\circ}$ line emission, we have used the criterion of the best morphological coincidence between the CS and the $\mathrm{Fe}^{\circ}$ line emissions. We have used the CS emission because, with a 


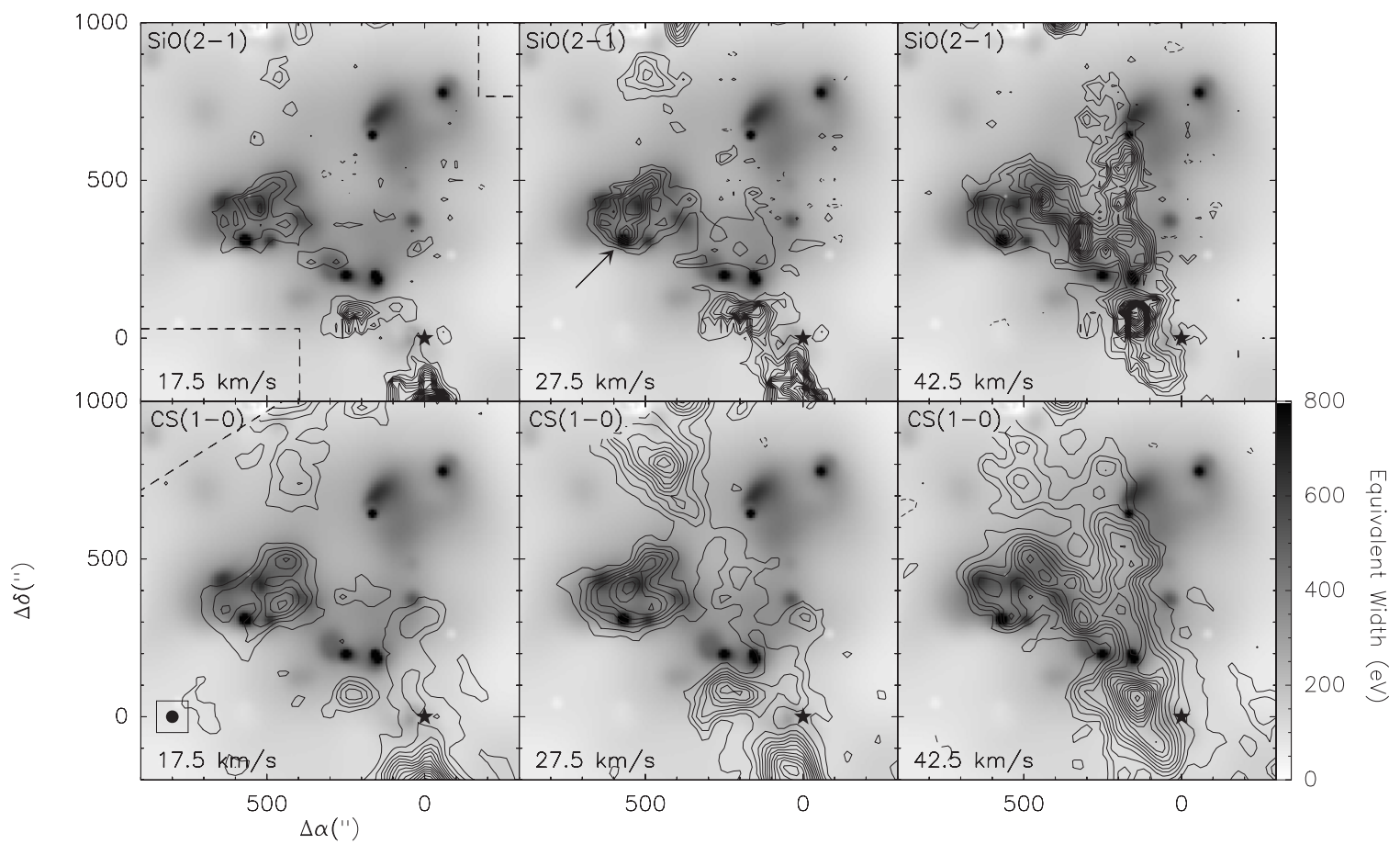

Figure 2. Same as Figure 1, but for the positive velocity channel maps. The black arrow in the $\mathrm{SiO}(2-1)$ panel with velocity $27.5 \mathrm{~km} \mathrm{~s}^{-1}$ points to the Park et al. (2004) knot (see Section 6.3).

critical density of $n_{c} \sim 10^{4} \mathrm{~cm}^{-3}$, it is one of the best tracers of high density gas (avoiding contamination from the diffuse gas of the galactic Disk along the line of sight), it is moderately affected by shocks (Requena-Torres et al. 2006), and it seems to survive UV radiation fields (Martín et al. 2008). Moreover, X-ray and far-UV fields are expected to slightly enhance its abundance in the gas phase (Stäuber et al. 2005). Therefore, we expect that CS emission will trace the large column densities of neutral material required to produce the $\mathrm{Fe}^{\circ}$ line emission, as was suggested by Martín-Pintado et al. (2000). We have found six velocity channels in the CS maps (whose widths are $\sim 5 \mathrm{~km} \mathrm{~s}^{-1}$ ) in which the CS distribution meets the morphological criterion of a match with the $\mathrm{Fe}^{\circ}$ line emission. Three of them correspond to the $-30 \mathrm{~km} \mathrm{~s}^{-1}$ molecular cloud (Serabyn \& Güsten 1987) with central velocities of $-22.5,-17.5$, and $-12.5 \mathrm{~km} \mathrm{~s}^{-1}$ (Figure 1), and the other three have radial velocities of $17.5,27.5$, and $42.5 \mathrm{~km} \mathrm{~s}^{-1}$ (Figure 2) and are associated with the G $0.11-0.11$ molecular cloud (see Tsuboi et al. 1997). The velocity channels presented in Figures 1 and 2 illustrate very well the spatial coincidences between the $\mathrm{CS}$ and the $\mathrm{Fe}^{\circ}$ emissions. The CS emission at the negative velocity channels (Figure 1) is associated with the Arched Thermal Filaments and, in general, follows the $\mathrm{Fe}^{\circ}$ line emission. The $27.5 \mathrm{~km} \mathrm{~s}^{-1} \mathrm{CS}$ channel map of the G 0.11-0.11 molecular cloud (Figure 2) shows an excellent correlation with the $\mathrm{Fe}^{\circ}$ line emission as previously shown by Yusef-Zadeh et al. (2002) and Koyama et al. (2003).

\section{THE $\mathrm{SiO} / \mathrm{CS}$ CORRELATION WITH THE $\mathrm{Fe}^{\circ}$ LINE}

For the comparison of the $\mathrm{SiO}$ and the CS emissions, we have smoothed and resampled our $\mathrm{SiO}$ data to the same velocity resolution as the CS maps, $5 \mathrm{~km} \mathrm{~s}^{-1}$. In the upper panels of Figures 1 and 2 one can see that the spatial distribution of the $\mathrm{SiO}$ emission does not completely follow that of the CS emission, but is more concentrated toward the peaks of the $\mathrm{Fe}^{\circ}$ line emission. The difference between the $\mathrm{CS}$ and the $\mathrm{SiO}$

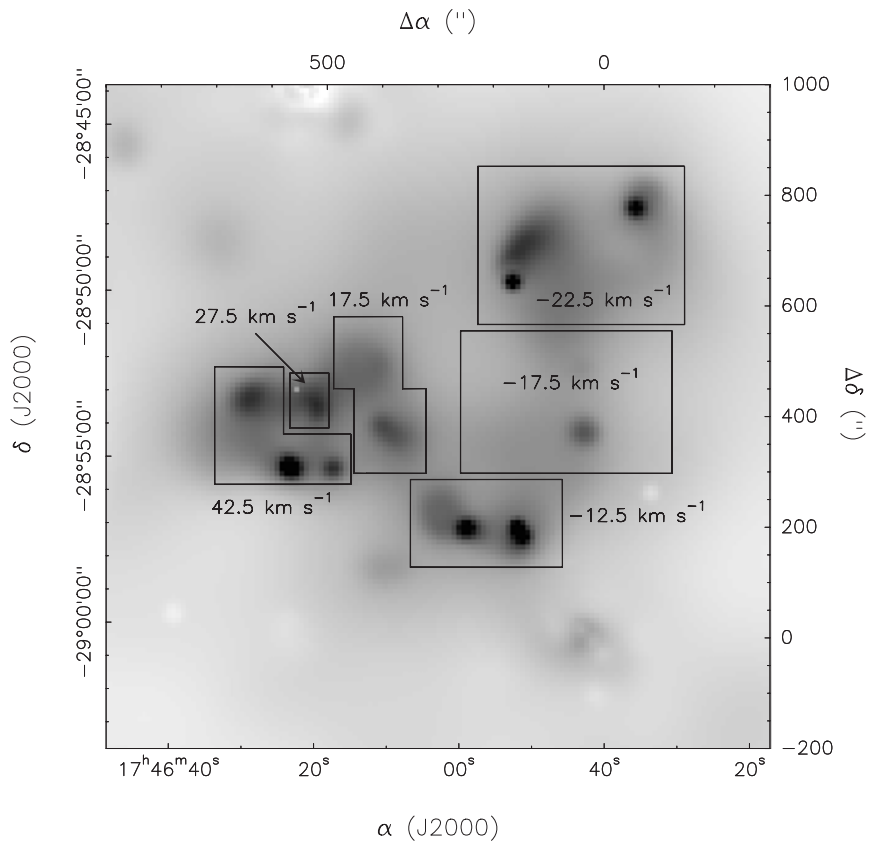

Figure 3. Image of the $\mathrm{Fe}^{\circ} \mathrm{EW}$ map. Polygons surround the regions where we have derived the $\mathrm{SiO} / \mathrm{CS}$ ratios that we represent in Figure 4. The numbers inside or near the polygons are the selected velocities used for the determination of the ratios in each region.

emission is clearly illustrated for the channel maps with negative velocities (Figure 1), where the CS emission is very prominent in the western region of the $-30 \mathrm{~km} \mathrm{~s}^{-1}$ molecular cloud, but neither $\mathrm{SiO}$ nor $\mathrm{Fe}^{\circ}$ line emission is detected there.

In order to establish whether the $\mathrm{SiO}$ abundance is enhanced in the molecular clouds with strong $\mathrm{Fe}^{\circ}$ line emission, we have calculated the $\mathrm{SiO} / \mathrm{CS}$ line intensity ratio for all selected velocity channels (which were previously spatially resampled 

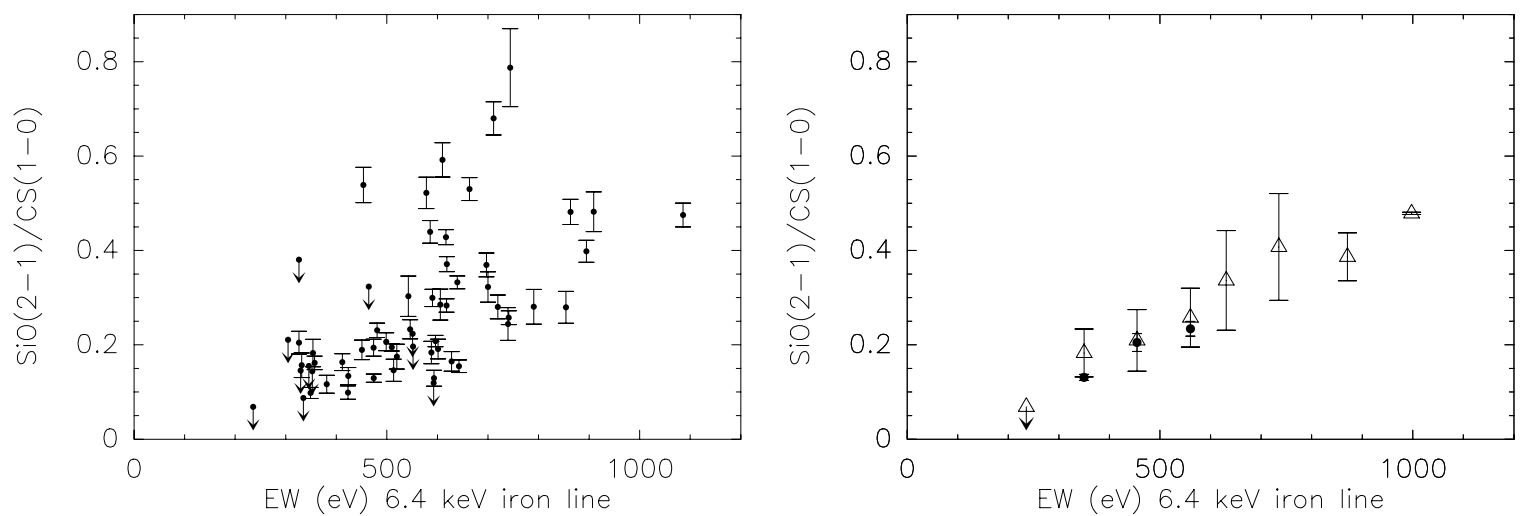

Figure 4. Correlation between the $\mathrm{SiO}(2-1) / \mathrm{CS}(1-0)$ line intensity ratios and the EW of the $\mathrm{Fe}^{\circ}$ line in the Sgr A XDR. Left: the circles show the data for single positions. The errorbars were derived from $1 \sigma$ noise level in $\mathrm{SiO}$ and $\mathrm{CS}$ spectra. Right: the open triangles represent the average value in bins of $100 \mathrm{eV}$, except for the two points with the highest EW, which were averaged together. The $1 \sigma$ standard deviation of the averaged data is represented by the errorbars. We have explored the effect of taking into account the upper limits on the average values using the maximum likelihood Kaplan-Meier estimator of the ratio in the three bins that present upper limits. For that purpose, the ASURV code was used (Feigelson \& Nelson 1985). The results with their associated uncertainties are represented by the filled circles.

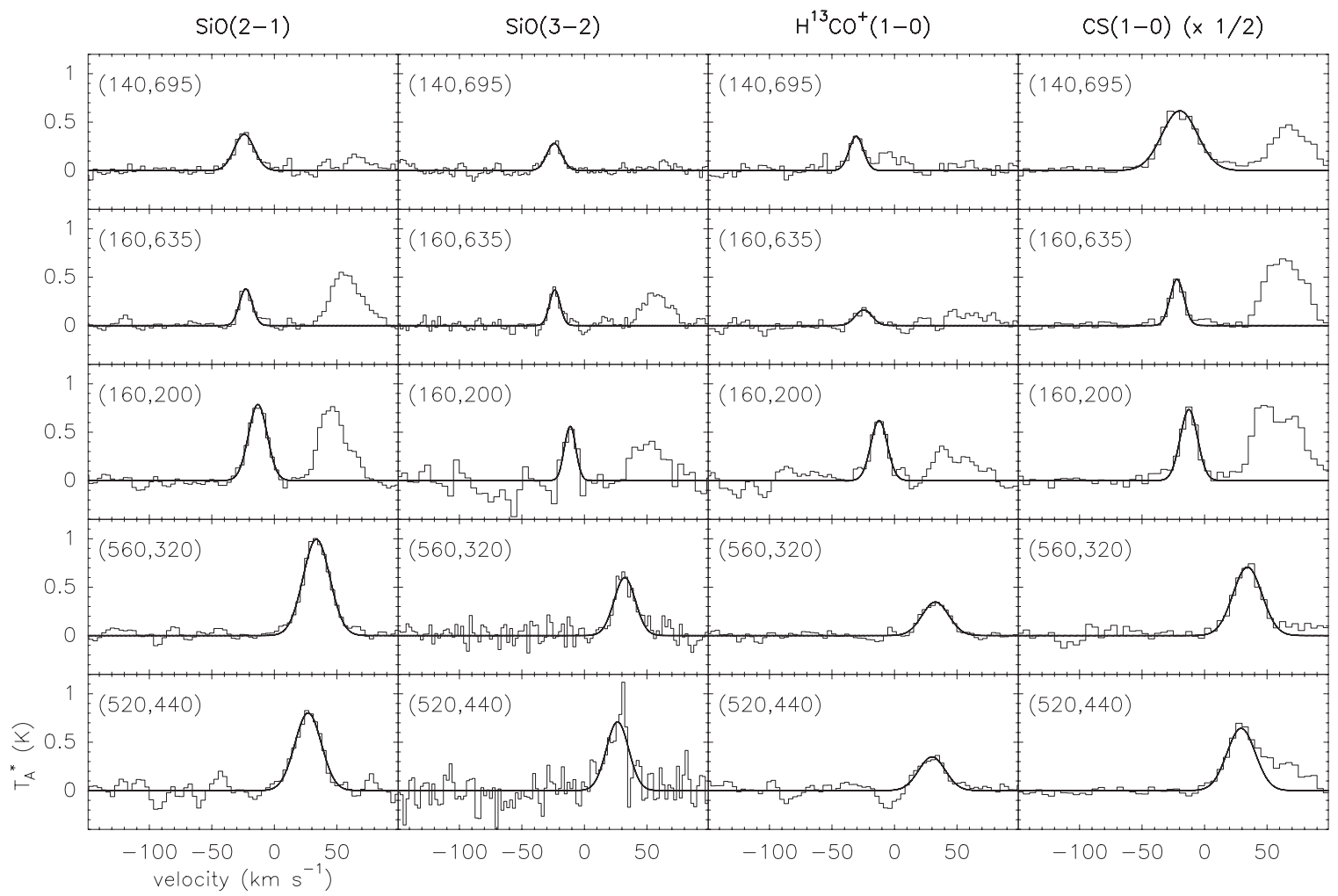

Figure 5. Spectra of $\mathrm{SiO}(2-1), \mathrm{SiO}(3-2), \mathrm{H}^{13} \mathrm{CO}^{+}(1-0)$, and $\mathrm{CS}(1-0)$ transitions for selected positions (white circles and ellipses in the bottom left panel of Figure 1) coincident with maxima of the $\mathrm{Fe}^{\circ}$ line intensity. In the upper left corner of each spectrum, we show its offset relative to the Sgr A* position. The spectra with central offsets $(140,695)$ and $(160,635)$ were obtained by averaging the spectra inside the regions shown in Figure 1 (white ellipses). The other three spectra result from single observations. CS(1-0) spectra were extracted from a data cube at the offset positions. We show the Gaussian fits only for the velocity components that have the best spatial correlation with the intensity of the $\mathrm{Fe}^{\circ}$ line. The Gaussian-fit parameters of the spectra are shown in Table 1.

to the same number of pixels. See Figure 3 to know which velocity channels correspond to which regions). The left panel of Figure 4 shows the $\mathrm{SiO} / \mathrm{CS}$ line intensity ratio as a function of the EW of the $\mathrm{Fe}^{\circ}$ line. First, we have spatially resampled the $\mathrm{Fe}^{\circ} \mathrm{EW}$ map to the same number of pixels than the molecular emission maps. Then, for the pixels with $\mathrm{Fe}^{\circ}$ line intensities greater than $500 \mathrm{eV}$, we have determined the $\mathrm{SiO} / \mathrm{CS}$ ratio from one pixel. For the regions where the $\mathrm{Fe}^{\circ}$ line emission is more diffuse, we have averaged over 9,20 , and 28 pixels depending on the strength of the CS emission. The error bars in the left panel of Figure $4(1 \sigma)$ were derived by propagating the rms noise of the $\mathrm{CS}$ and $\mathrm{SiO}$ spectra. Points with a vertical arrow represent upper limits to the $\mathrm{SiO}$ emission at a $3 \sigma$ level.

As observed by Martín-Pintado et al. (2000) for the Sgr B complex, we find a systematic increase of the $\mathrm{SiO} / \mathrm{CS}$ line ratio as a function of the strength of the $\mathrm{Fe}^{\circ}$ line. The $\mathrm{SiO} / \mathrm{CS}$ ratio increases by more than one order of magnitude when the $\mathrm{Fe}^{\circ} \mathrm{EW}$ changes from 200 to $700 \mathrm{eV}$. There is, however, a substantial dispersion in the $\mathrm{SiO} / \mathrm{CS}$ ratio when the $\mathrm{EW}$ is larger than $500 \mathrm{eV}$. In fact, the largest $\mathrm{SiO} / \mathrm{CS}$ ratio of $\sim 0.8$ is not found for the largest $\mathrm{Fe}^{\circ}$ EWs. The data seem to show a bimodal distribution with two sets of data points bifurcating at $\sim 650 \mathrm{eV}$. 
Table 1

Gaussian-fit Parameters of the Spectra Shown in Figure 5

\begin{tabular}{|c|c|c|c|c|c|}
\hline $\begin{array}{l}\text { Offset } \\
\left({ }^{\prime \prime}\right)\end{array}$ & Transition & $\begin{array}{c}\int T_{A}^{*} d v \\
\left(\mathrm{~K} \mathrm{~km} \mathrm{~s}^{-1}\right)\end{array}$ & $\begin{array}{c}v_{0} \\
\left(\mathrm{~km} \mathrm{~s}^{-1}\right) \\
\end{array}$ & $\begin{array}{c}\Delta v \\
\left(\mathrm{~km} \mathrm{~s}^{-1}\right)\end{array}$ & $\begin{array}{c}I_{0} \\
(\mathrm{~K}) \\
\end{array}$ \\
\hline \multirow[t]{4}{*}{$(140,695)$} & $\mathrm{SiO}(2-1)$ & $7.5 \pm 0.5$ & $-24.1 \pm 0.6$ & $18.8 \pm 1.7$ & $0.37 \pm 0.04$ \\
\hline & $\mathrm{SiO}(3-2)$ & $4.2 \pm 0.4$ & $-24.1 \pm 0.7$ & $14.1 \pm 1.7$ & $0.28 \pm 0.05$ \\
\hline & $\mathrm{H}^{13} \mathrm{CO}^{+}(1-0)$ & $5.0 \pm 0.5$ & $-30.6 \pm 0.6$ & $13.3 \pm 1.6$ & $0.35 \pm 0.04$ \\
\hline & $\operatorname{CS}(1-0)^{\mathrm{a}}$ & $20.1 \pm 0.6$ & -24 & {$[-33,-15]$} & $1.22 \pm 0.06$ \\
\hline \multirow[t]{4}{*}{$(160,635)$} & $\mathrm{SiO}(2-1)$ & $4.8 \pm 0.4$ & $-22.8 \pm 0.5$ & $12.0 \pm 1.3$ & $0.38 \pm 0.04$ \\
\hline & $\mathrm{SiO}(3-2)$ & $3.9 \pm 0.3$ & $-23.7 \pm 0.4$ & $10.1 \pm 1.1$ & $0.37 \pm 0.04$ \\
\hline & $\mathrm{H}^{13} \mathrm{CO}^{+}(1-0)$ & $2.7 \pm 0.4$ & $-24.6 \pm 1.3$ & $16 \pm 3$ & $0.16 \pm 0.04$ \\
\hline & $\mathrm{CS}(1-0)$ & $13.2 \pm 0.8$ & $-22.0 \pm 0.4$ & $13 \pm 1$ & $0.96 \pm 0.06$ \\
\hline \multirow[t]{4}{*}{$(160,200)$} & $\mathrm{SiO}(2-1)$ & $14.8 \pm 0.9$ & $-13.2 \pm 0.5$ & $17.6 \pm 1.2$ & $0.79 \pm 0.07$ \\
\hline & $\mathrm{SiO}(3-2)$ & $6.4 \pm 1.1$ & $-11.5 \pm 1.0$ & $10.5 \pm 1.7$ & $0.57 \pm 0.11$ \\
\hline & $\mathrm{H}^{13} \mathrm{CO}^{+}(1-0)$ & $10.2 \pm 0.9$ & $-12.3 \pm 0.6$ & $15.5 \pm 1.7$ & $0.62 \pm 0.07$ \\
\hline & $\mathrm{CS}(1-0)$ & $25.4 \pm 1.2$ & $-12.5 \pm 0.4$ & $16 \pm 1$ & $1.47 \pm 0.09$ \\
\hline \multirow[t]{4}{*}{$(560,320)$} & $\mathrm{SiO}(2-1)$ & $26.7 \pm 0.7$ & $33.7 \pm 0.3$ & $25.2 \pm 0.8$ & $0.99 \pm 0.05$ \\
\hline & $\mathrm{SiO}(3-2)$ & $12.8 \pm 1.0$ & $32.2 \pm 0.8$ & $20.0 \pm 2.1$ & $0.60 \pm 0.10$ \\
\hline & $\mathrm{H}^{13} \mathrm{CO}^{+}(1-0)$ & $9.2 \pm 0.6$ & $32.8 \pm 0.9$ & $25.0 \pm 1.9$ & $0.35 \pm 0.05$ \\
\hline & $\mathrm{CS}(1-0)$ & $41.1 \pm 2.1$ & $34.3 \pm 0.7$ & $27.3 \pm 1.7$ & $1.41 \pm 0.12$ \\
\hline \multirow[t]{4}{*}{$(520,440)$} & $\mathrm{SiO}(2-1)$ & $21.3 \pm 0.9$ & $27.0 \pm 0.5$ & $24.9 \pm 1.3$ & $0.80 \pm 0.07$ \\
\hline & $\mathrm{SiO}(3-2)$ & $15.9 \pm 2.0$ & $26.5 \pm 1.2$ & $21 \pm 4$ & $0.71 \pm 0.17$ \\
\hline & $\mathrm{H}^{13} \mathrm{CO}^{+}(1-0)$ & $9.3 \pm 0.9$ & $30.0 \pm 1.2$ & $25 \pm 3$ & $0.35 \pm 0.07$ \\
\hline & $\mathrm{CS}(1-0)$ & $36 \pm 4$ & $29.3 \pm 1.1$ & $26.6 \pm 2.1$ & $1.29 \pm 0.07$ \\
\hline
\end{tabular}

Notes.

${ }^{a}$ In this case, we do not present the results of the Gaussian fit because the resulting width was twice the width of the other lines (there was $\mathrm{CS}$ emission with no $\mathrm{SiO}$ and $\mathrm{H}^{13} \mathrm{CO}^{+}$counterpart). Therefore, in order to derive the $\mathrm{CS}$ column density in Table 2, we have integrated only between the velocities where the $\mathrm{SiO}(2-1)$ emission arises, which is the velocity interval shown in the 5th column.

It is interesting to note that all the points in the data set with the highest $\mathrm{SiO} / \mathrm{CS}$ ratio that seem to follow a steeper slope are located in the eastern region of the $\mathrm{G} 0.11-0.11$ molecular cloud, where Tsuboi et al. (1997) proposed that an interaction of the cloud with the intense magnetic field traced by the Radio Arc could be taking place. One should stress that, in spite of the $\mathrm{SiO} / \mathrm{CS}$ dispersion, the region defined by the lowest $\mathrm{SiO} / \mathrm{CS}$ ratios also clearly shows a systematic trend of increasing with $\mathrm{Fe}^{\circ} \mathrm{EW}$.

The right panel of Figure 4 shows, as open triangles, the averages of all the individual values of the $\mathrm{SiO} / \mathrm{CS}$ ratio when binned to $100 \mathrm{eV}$, except for the two points with the highest $\mathrm{EW}$, which have been averaged together. The error bars represent the standard deviation of the averaged value. This figure shows a good correlation between the $\mathrm{Fe}^{\circ}$ line and the averaged $\mathrm{SiO} / \mathrm{CS}$ abundance ratio.

As discussed by Martín-Pintado et al. (2000), the SiO/CS line ratios should provide a very good approximation of the column density ratios, since $\mathrm{SiO}$ and $\mathrm{CS}$ have similar dipole moments and energy level distributions. Then, the $\mathrm{SiO} / \mathrm{CS}$ line ratios do not depend strongly on the physical conditions if the emission is optically thin.

\section{PHYSICAL CONDITIONS OF THE GAS}

In order to evaluate the physical conditions of the gas, we selected five positions at the $\mathrm{Fe}^{\circ}$ line intensity peaks, shown with white circles (individual spectra) and ellipses (averaged spectra) in the bottom left panel of Figure 1. In Figure 5 we show the spectra of the $J=2 \rightarrow 1$ and $J=3 \rightarrow 2$ transitions of $\mathrm{SiO}$, and the $J=1 \rightarrow 0$ lines of $\mathrm{H}^{13} \mathrm{CO}^{+}$and $\mathrm{CS}$, together with the Gaussian fits of the velocity components that show the best morphological correlation with the X-ray emission. The fitted parameters are tabulated in Table 1.

To derive the excitation temperature $\left(T_{\mathrm{ex}}\right)$, opacity $(\tau)$, and column density of the $\mathrm{SiO}$ emission, as well as the volume density $\left(n_{\mathrm{H}_{2}}\right)$ of the medium, we have fitted both transitions of $\mathrm{SiO}$ using a large velocity gradient (LVG) excitation code. From a survey of ammonia inversion lines toward several molecular clouds near the GC, Hüttemeister et al. (1993) derived the presence of a kinetic temperature gradient in the clouds, with gas temperatures varying from $\sim 25$ to $200 \mathrm{~K}$. Rodríguez-Fernández et al. (2001), from $\mathrm{S}(0)$ to $\mathrm{S}(5) \mathrm{H}_{2}$ pure rotational lines, also found a temperature gradient in the GC molecular clouds, with an average fraction of warm gas $(\sim 150 \mathrm{~K})$ of $\sim 30 \%$. Therefore, a kinetic temperature of $\sim 70 \mathrm{~K}$ should account for at least half of the total $\mathrm{H}_{2}$ column density. Handa et al. (2006) also adopted this value in their estimation of the $\mathrm{H}_{2}$ column density in the E ridge of the $\mathrm{G} 0.11-0.11$ molecular cloud (our (520, $440)^{\prime \prime}$ position). The results from the LVG model for the five selected positions are shown in Table 2 . The excitation is clearly subthermal $\left(T_{\mathrm{ex}} \ll T_{K}\right)$, the emission is optically thin $(\tau<1)$, and the $\mathrm{SiO}$ column densities range from 0.8 to $6 \times 10^{13} \mathrm{~cm}^{-2}$. We have also derived the $\mathrm{H}^{13} \mathrm{CO}^{+}$and $\mathrm{CS}$ column densities $\left(N_{\mathrm{H}^{13} \mathrm{CO}^{+}}\right.$and $\left.N_{\mathrm{CS}}\right)$ applying the $\mathrm{LVG}$ model, with the physical conditions derived from the $\mathrm{SiO}$ transitions. $N_{\mathrm{H}^{13} \mathrm{CO}^{+}}$ranges between 2 and $8 \times 10^{12} \mathrm{~cm}^{-2}$, while $N_{\mathrm{CS}}$ ranges from 1 to $3 \times 10^{14} \mathrm{~cm}^{-2}$.

In order to evaluate the uncertainty in our derived values from the LVG analysis due to the errors in the line intensities, we have taken into account the Gaussian-fit errors and a pessimistic calibration error of $20 \%$ for all the transitions. Then, we have run the LVG code for all the possible range of the line intensities $( \pm 1 \sigma)$. We have also considered in our analysis the possible extreme values of the kinetic temperature ( 25 and $200 \mathrm{~K})$. In all the cases, the excitation temperatures are clearly subthermal, ranging from 4 to $13 \mathrm{~K}$, the emission is optically thin, and the error in the $\mathrm{SiO}$ column density is less than a factor of 2, showing almost no variation with kinetic temperature. The $\mathrm{H}_{2}$ volume density changes by a factor of 4 , however, this error does not translate into a large dispersion in the $\mathrm{H}^{13} \mathrm{CO}^{+}$column 
Table 2

Physical Conditions of the Gas Derived from the Spectra Shown in Figure 5

\begin{tabular}{|c|c|c|c|c|c|c|c|c|}
\hline $\begin{array}{l}\text { Offset } \\
\left({ }^{\prime \prime}\right)\end{array}$ & $\begin{array}{c}N_{\mathrm{SiO}} \\
\left(\times 10^{13} \mathrm{~cm}^{-2}\right)\end{array}$ & $\tau$ & $\begin{array}{l}T_{\mathrm{ex}} \\
(\mathrm{K})\end{array}$ & $\begin{array}{c}n_{\mathrm{H}_{2}} \\
\left(\times 10^{5} \mathrm{~cm}^{-3}\right)\end{array}$ & $\begin{array}{c}N_{\mathrm{H}^{13} \mathrm{CO}^{+}} \\
\left(\times 10^{12} \mathrm{~cm}^{-2}\right)\end{array}$ & $\begin{array}{c}N_{\mathrm{H}_{2}} \\
\left(\times 10^{22} \mathrm{~cm}^{-2}\right)\end{array}$ & $\begin{array}{c}N_{\mathrm{CS}} \\
\left(\times 10^{14} \mathrm{~cm}^{-2}\right)\end{array}$ & $\begin{array}{c}N_{\mathrm{H}_{2}} \\
\left(\times 10^{22} \mathrm{~cm}^{-2}\right)\end{array}$ \\
\hline$(140,695)$ & 1.0 & 0.1 & 6 & 1 & 4 & 0.8 & 2 & 2 \\
\hline$(160,635)$ & 0.8 & 0.08 & 8 & 2 & 2 & 0.4 & 1 & 1 \\
\hline$(160,200)$ & 2 & 0.3 & 6 & 1 & 8 & 1.6 & 2 & 2 \\
\hline$(560,320)$ & 6 & 0.4 & 5 & 0.6 & 7 & 1.4 & 2 & 2 \\
\hline$(520,440)$ & 3 & 0.2 & 7 & 2 & 7 & 1.4 & 3 & 3 \\
\hline
\end{tabular}

Notes. Physical conditions and molecular abundances in five selected positions (offsets are shown in arcseconds in Column 1). We derived the $\mathrm{SiO}$ column density (Column 2), opacity (Column 3), excitation temperature (Column 4), and volume density (Column 5) of the medium fitting

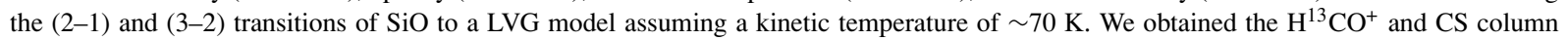
densities (Columns 6 and 8) fitting their (1-0) transition to a LVG with the physical conditions derived from SiO transitions. The molecular hydrogen column densities in Columns 7 and 9 were derived from the $\mathrm{H}^{13} \mathrm{CO}^{+}$and CS column densities, respectively. See Section 5 for more details.

densities. The uncertainty due to the allowed range of solutions for the $\mathrm{H}_{2}$ volume density and to the errors of the $\mathrm{H}^{13} \mathrm{CO}^{+}(1-0)$ line intensity is less than a factor of 2 . Moreover, the $\mathrm{H}^{13} \mathrm{CO}^{+}$ column density does not show substantial variations for the kinetic temperatures between 25 and $200 \mathrm{~K}$.

Taking into account the isotopic ratio ${ }^{12} \mathrm{C} /{ }^{13} \mathrm{C}$ for the $\mathrm{GC}$ of $\sim 20$ (Wilson \& Rood 1994), and a fractional abundance $X_{\mathrm{HCO}^{+}}=N_{\mathrm{HCO}^{+}} / N_{\mathrm{H}_{2}} \sim 10^{-8}$ (Irvine et al. 1987), we derive $N_{\mathrm{H}_{2}} \sim(0.4-1.6) \times 10^{22} \mathrm{~cm}^{-2}$ (Column 7 of Table 2). These $\mathrm{H}_{2}$ column densities are more than one order of magnitude lower than the column densities derived by Handa et al. (2006). This discrepancy can be attributed to the assumed fractional abundance of $\mathrm{H}^{13} \mathrm{CO}^{+}$by Handa et al. (2006) of $X_{\mathrm{H}^{13} \mathrm{CO}^{+}} \sim$ $10^{-10}$ derived using a ${ }^{12} \mathrm{C} /{ }^{13} \mathrm{C}$ isotopic ratio of 90 (the one used by García-Burillo et al. (2000) for NGC 253), together with the assumption that the $\mathrm{H}^{13} \mathrm{CO}^{+}(1-0)$ emission is thermalized at the GC densities, whereas our LVG analysis shows this transition to be subthermally excited for $n_{\mathrm{H}_{2}} \sim(0.6-2) \times 10^{5} \mathrm{~cm}^{-3}$. The column densities $N_{\mathrm{H}_{2}} \sim(1-3) \times 10^{22} \mathrm{~cm}^{-2}$ derived from CS emission by assuming a fractional abundance $X_{\mathrm{CS}} \sim 10^{-8}$ (Irvine et al. 1987) are similar, within a factor of $2-3$, to those we derived from $\mathrm{H}^{13} \mathrm{CO}^{+}$.

\section{DISCUSSION}

The spatial variations of the $\mathrm{SiO} / \mathrm{CS}$ ratios could be due to variations in the abundances of $\mathrm{CS}, \mathrm{SiO}$ or in both. However, the CS abundance seems to be rather uniform in the GC clouds, independent of the type of dominating chemistry. The CS abundance does not seem to be very affected by either the ejection of molecules from grain mantles (Requena-Torres et al. 2006) or by photodissociation (Martín et al. 2008). The changes in the $\mathrm{SiO} / \mathrm{CS}$ line ratio with the $\mathrm{Fe}^{\circ}$ line intensity suggest that the $\mathrm{SiO}$ abundance could be enhanced by the same mechanism that also give rise to the $\mathrm{Fe}^{\circ}$ line. So far, the origin of the $\mathrm{Fe}^{\circ}$ line is subject to debate (Koyama et al. 2008; Yusef-Zadeh et al. 2007). In the following, we discuss the different mechanisms that could explain the observed correlation between the $\mathrm{SiO} /$ $\mathrm{CS}$ line intensity ratio and the $\mathrm{Fe}^{\circ}$ line.

\subsection{X-ray Reflection Nebula: Grain Destruction}

In the X-ray reflection nebula (XRN) scenario, the $\mathrm{Fe}^{\circ}$ emission is a consequence of the illumination of the GC molecular clouds by external X-ray sources, together with Thomson scattering and/or absorption of these X-rays by the gas and dust in the clouds (Koyama et al. 1996; Sunyaev \& Churazov 1998; Murakami et al. 2000, 2001). This scenario explains the weak hard X-ray continuum emission and the strong $\mathrm{Fe}^{\circ}$ line, produced by a past episode of bright X-ray emission from some source in the GC, possibly the central supermassive black hole, Sgr A*. This model has been applied to three of the most massive molecular clouds in the GC region: Sgr B2 (Murakami et al. 2000), Sgr C (Murakami et al. 2001) and the $\mathrm{G} 0.11-0.11$ molecular cloud, also called M $0.11-0.08$ or G 0.13-0.13 (Murakami et al. 2003). Park et al. (2004) have also proposed that the peak $\mathrm{Fe}^{\circ}$ emission in several knots could be compatible with the XRN scenario, although other mechanisms like SNRs cannot be ruled out for some of the $\mathrm{Fe}^{\circ}$ line emission (see Section 6.3).

According to this hypothesis, the enhancement of the $\mathrm{SiO}$ abundance could be due to the interaction of hard X-ray photons $(E>7.1 \mathrm{keV})$ with gas and dust grains. The photoelectrons produced by the X-rays will deposit their energy while they traverse the grains, giving rise to temperature fluctuations. In the case of small grain sizes, $\sim 10 \AA$, the peak temperature could be as high as $1000 \mathrm{~K}$, making possible the evaporation of grains, which will release atomic $\mathrm{Si}$ or $\mathrm{SiO}$ to the gas phase. The fraction of Si atoms evaporated from one grain could be as high as $0.1 \%$ (Voit 1991; Martín-Pintado et al. 2000).

However, to produce the observed $\mathrm{SiO}$ abundance enhancements, there must be a significant population of very small silicate grains. Theory predicts the existence of such a population of small grains in environments where shocks play an important role, due to the redistribution of the grain sizes through shattering (grain-grain collisions; Jones et al. 1996). In the GC, where the widespread $\mathrm{SiO}$ emission is attributable to shocks (Martín-Pintado et al. 1997; Requena-Torres et al. 2006), the presence of a population of silicate nanograins is very likely. The non-detection of the $9.7 \mu \mathrm{m}$ emission feature constrains the fraction of silicate mass depleted onto very small grains to $\mathrm{Y}_{s} \leqslant 1 \%-5 \%$ (Desert et al. 1986; Siebenmorgen et al. 1992). However, this upper limit could be higher, as Li \& Draine (2001) have shown in the diffuse interstellar medium $(10 \%$ for grains with $a \leqslant 15 \AA$ ).

From our estimations of $N_{\mathrm{H}_{2}}$ and $N_{\mathrm{SiO}}$ at the five positions of the maxima in the $\mathrm{X}$-ray emission (Table 2), we derive a $\mathrm{SiO}$ fractional abundance of $\sim 10^{-9}$ (in agreement with previous estimations in the GC: Hüttemeister et al. 1998; Martín-Pintado et al. 1997, 2000; Rodríguez-Fernández et al. 2006). Yan (1997) gives a value for the elemental abundance of silicon: $X_{\mathrm{Si}}=n_{\mathrm{Si}} / n_{H} \sim 3.55 \times 10^{-5}$. Considering that the metallicity at the GC is likely twice the solar value (Morris \& Serabyn $1996)$, that $[\mathrm{H} \mathrm{I}] /\left[\mathrm{H}_{2}\right] \sim 0.05$ in the central $500 \mathrm{pc}$ of the 
Galaxy (Güsten \& Philipp 2004), and assuming that most of the $\mathrm{Si}$ is ejected from grains in the form of $\mathrm{SiO}$, we conclude that with only a small fraction of atoms evaporated from small grains $(\sim 0.01 \%)$, we could reproduce the observed fractional abundances.

Another mechanism, proposed by Mitchell et al. (2002), that could help to disrupt small grains $(\sim 100 \AA)$ in the presence of hard X-rays is fragmentation induced by Coulomb forces. The ejection of a photoelectron after the absorption of an X-ray photon by an atom, creates a hole in an electron level that can be filled by an electronic transition from an upper level. This transition can occur by a radiative process (fluorescence) or by the ejection of a second electron from some energetically allowed level (the Auger effect). This process can continue leading to an Auger cascade. According to Mitchell et al. (2002), in the case of fluffy aggregated grains composed of particles $\sim 100 \AA$ in size irradiated by hard X-rays photons, some of the Auger electrons will not be able to escape from the grain (most of the Auger electrons generated in $\mathrm{Si}, \mathrm{Mg}$, and $\mathrm{O}$ atoms have energies $\leqslant 1 \mathrm{keV}$, whose mean free path in a silicate grain with density $3.3 \mathrm{~g} \mathrm{~cm}^{-3}$ is $\leqslant 220 \AA \AA$; Dwek \& Smith 1996; Voit 1991). Therefore, they will undergo further collisions with other atoms of the aggregate resulting in secondary electron emission, which will leave the particles with net positive charge. This positive charge will give rise to a small electric potential, but taking into account the small size of the particles, this potential will translate into a huge electric field that could generate emission of tertiary electrons from other particles in the grain, causing further charge buildup. If the electrostatic stress induced in the particles due to charging exceed the tensile strength of the material, fragmentation of the grain will occur (Mitchell et al. 2002). This could be likely for aggregated grains composed of particles with sizes $\leqslant 100 \AA$ A. This kind of grain could be present in regions where a significant population of small grains exists.

\subsection{X-ray Reflection Nebula: Gas-phase Chemistry}

Another possibility to explain the $\mathrm{SiO}$ enhancement is gasphase chemistry driven by X-rays. To check this possibility, we have made a comparison with the XDR models of Meijerink \& Spaans (2005). The two input parameters in these models are volume density $\left(n_{H}=n_{\mathrm{H}_{1}}+2 n_{\mathrm{H}_{2}} \approx 2 n_{\mathrm{H}_{2}}\right.$ for the GC; Güsten \& Philipp 2004) and X-ray flux $\left(F_{X}\right)$. Once both parameters have been fixed, we can predict the $\mathrm{SiO}(2-1) / \mathrm{CS}(1-0)$ ratio for different values of the column density $\left(N_{\mathrm{H}}\right)$ affected by the $\mathrm{X}$-rays.

For the column and volume densities derived in Section 5 $\left(N_{\mathrm{H}} \sim(0.7-3) \times 10^{22} \mathrm{~cm}^{-2}\right.$ and $n_{\mathrm{H}} \sim(1.2-4) \times 10^{5} \mathrm{~cm}^{-3}$, respectively), the XDR model that could account for the observed $\mathrm{SiO}(2-1) / \mathrm{CS}(1-0)$ line intensity ratios at the five selected positions of Table 2 requires X-ray fluxes of $F_{X}=1.6 \mathrm{erg} \mathrm{cm}^{-2}$ $\mathrm{s}^{-1}$ (the lowest flux used by Meijerink \& Spaans (2005) in their models). Higher X-ray fluxes will predict line ratios larger than unity. According to Murakami et al. (2001), Sgr A* should have had a luminosity of $\sim 10^{39} \mathrm{erg} \mathrm{s}^{-1}$ about $300 \mathrm{yr}$ ago to explain the $\mathrm{Fe}^{\circ}$ line intensities observed for the Sgr C and Sgr B2 complexes. In that case, we would have an X-ray flux of $F_{X} \sim(2.2-0.12) \times 10^{-1} \mathrm{erg} \mathrm{cm}^{-2} \mathrm{~s}^{-1}$ (using the projected distance) in the regions of strong $\mathrm{Fe}^{\circ}$ line emission closer to and further from Sgr A*, respectively, in the Sgr A complex.

Therefore, for the values we derive for $n_{\mathrm{H}_{2}}$ and $N_{\mathrm{H}_{2}}$, and the XDR models of Meijerink \& Spaans (2005), we reach the conclusion that the chemistry induced by X-rays could explain the $\mathrm{SiO}$ enhancement only if the luminosity of $\mathrm{Sgr} \mathrm{A}^{*} 300 \mathrm{yr}$ ago would had been one or two orders of magnitude higher than the one derived from the XRN model, i.e.; $\sim 10^{40-41} \mathrm{erg} \mathrm{s}^{-1}$.

However, strong support for the XRN scenario comes from the variability in the intensity and morphology of the $4-8 \mathrm{keV}$ X-ray emission that Muno et al. (2007) have reported in the Sgr A complex. Recently, this variability has been confirmed by Koyama et al. (2009) from the Chandra (2002) to the Suzaku (2005) observations. Muno et al. (2007) claimed that the variability clearly supports the XRN origin of the $\mathrm{Fe}^{\circ}$ line emission, as matter (low energy electrons or ejecta blobs from supernovae $(\mathrm{SNe})$, which are the other candidates to produce the $\mathrm{Fe}^{\circ}$ emission; see Section 6.3) would propagate too slowly to produce the observed variability in only three years. They reach the conclusion that the fluorescent emission must have its origin in a short and intense X-ray outburst of $\mathrm{Sgr}^{\mathrm{*}}{ }^{*}$ or some other accretion-powered X-ray source. In this case, the $\mathrm{SiO}$ abundance would be produced by evaporation from very small silicon grains.

\subsection{Low Energy Cosmic Rays (LECRs)}

Valinia et al. (2000) suggested that the $\mathrm{Fe}^{\circ}$ line emission could be explained by impact ionization of molecular gas by low-energy cosmic-ray electrons (LECRs) with energy of $\sim 30 \mathrm{keV}$. They proposed that the observed spectrum in X-rays could be fitted by a nonthermal component plus a thermal one originating in shocks, instead of two non-equilibrium-ionization thermal components with temperatures of $\sim 0.8$ and $7 \mathrm{keV}$. This hypothesis avoided two serious problems: the origin of the $7 \mathrm{keV}$ component (X-rays produced by SN shocks only reach $3 \mathrm{keV}$ ) and its confinement (the plasma exceeds the gravitational potential in the region, so the hot gas must be constantly and uniformly replenished; Belmont et al. 2005).

This model has been applied to the G 0.11-0.11 molecular cloud by Yusef-Zadeh et al. (2002), and has been recently extended to the rest of the dense clouds in the GC with $\mathrm{Fe}^{\circ}$ line emission (Yusef-Zadeh et al. 2007). Basically, Yusef-Zadeh et al. (2007) calculate the production rate of the $\mathrm{Fe}^{\circ}$ photons associated with the injection of electrons per unit energy interval and time, into a cloud with a given column density. Their predicted flux for the $\mathrm{Fe}^{\circ}$ line is one order of magnitude smaller than that observed for the Sgr A clouds near the Radio Arc. Only very large column densities of $\sim 10^{24} \mathrm{~cm}^{-2}$ could fit the observations. However, such a high column density for the molecular gas is incompatible with our estimates by nearly two orders of magnitude (see Table 2). In this scenario, the $\mathrm{SiO}$ enhancement could be produced by the same process that also accelerates the electrons, i.e., shocks generated by SNe (Bykov et al. 2002). Metal-rich ejecta blobs from an SN that propagates through the ambient medium create shocks, which accelerate the electrons. Internal shocks are also generated within the ejecta blobs. The population of high energy electrons will irradiate the ejecta blobs, generate the $\mathrm{Fe}^{\circ}$ line emission, and produce the non-thermal bremsstrahlung emission. As ions and electrons are accelerated to high energies, they will penetrate deep into the ejecta blobs enhancing the grain erosion. Also, the shocks propagating into the molecular cloud and into the ejecta blobs will sputter and erode the grains, ejecting $\mathrm{Si}$ atoms or $\mathrm{SiO}$ molecules to the gas phase.

Park et al. (2004) reported the presence of a soft thermal component in the spectrum of one knot (see arrow in Figure 2) that could indicate a contribution from SN ejecta. The angular 
size of this knot is $1^{\prime}$ or $2.5 \mathrm{pc}$ at the GC distance, which is compatible with the size of a typical young Galactic SNR or a relatively older SNR if it is propagating into a dense molecular cloud (Park et al. 2004). In fact, this knot appears in Figure 2 well correlated with the $\mathrm{SiO}$ emission, consistent with the $\mathrm{SNe}$ scenario.

Gray (1994) has found an excess of SNRs in the GC over that expected for a uniform Galaxy distribution. In the Sgr A complex, there are at least two identified SNRs: Sgr A East and G 359.92-0.09 (Maeda et al. 2002; Coil \& Ho 2000). However, at the expected interaction sites of these SNRs with the 50 and $20 \mathrm{~km} \mathrm{~s}^{-1}$ molecular clouds, respectively, the $\mathrm{Fe}^{\circ}$ line does not show any excess emission, although the $\mathrm{SiO} / \mathrm{CS}$ value is comparable to the highest values found in Figure 4. It is possible that these SNRs are too old to still have ejecta with sufficient energy to effectively accelerate electrons and produce the fluorescent emission, but grains might still be sputtered by shocks.

\section{CONCLUSIONS}

We have mapped the Sgr A region in the $J=2 \rightarrow 1$ line of $\mathrm{SiO}$, and have found that the $\mathrm{SiO} / \mathrm{CS}$ intensity ratio in the Sgr A region is correlated with the $\mathrm{EW}$ of the neutral or low-ionization $\mathrm{Fe} \mathrm{K} \alpha$ fluorescence line at $6.4 \mathrm{keV}$. The $\mathrm{SiO}$ abundance is enhanced by nearly one order of magnitude toward the regions with strong emission in the $6.4 \mathrm{keV} \mathrm{Fe}$ line. We have discussed the two mechanisms previously proposed in the literature to explain the $6.4 \mathrm{keV} \mathrm{Fe}$ line in the context of the $\mathrm{SiO}$ abundance enhancement found in this paper, namely the XRN and LECR scenarios.

The LECR scenario seems to explain the $\mathrm{SiO}$ enhancement in a natural way in the case that the electron acceleration has been produced in shocks associated with SNRs propagating through dense molecular clouds. The main weaknesses of this hypothesis are the high gas column density required to produce the flux of the $6.4 \mathrm{keV} \mathrm{Fe} \mathrm{line,} \mathrm{and} \mathrm{the} \mathrm{existence} \mathrm{of} \mathrm{molecular}$ clouds that show evidence of interaction with SNRs but which do not correlate with the $6.4 \mathrm{keV}$ Fe line intensity. In the XRN scenario, the $\mathrm{SiO}$ enhancement can only be explained by the evaporation of very small silicate grains, the presence of which is still under debate. The reported variability in the $6.4 \mathrm{keV} \mathrm{Fe}$ line intensity supports this scenario.

We thank Professor M. Tsuboi for kindly providing the CS $(1-0)$ data. This work has been supported by the Spanish Ministerio de Educación y Ciencia under projects ESP 200400665 and ESP2007-65812-C02-01 and the "Comunidad de Madrid" Government under PRICIT project S-0505/ESP-0237 (ASTROCAM).

\section{Facility: IRAM-30m, Pico Veleta, Granada (Spain)}

\section{REFERENCES}

Belmont, R., Tagger, M., Muno, M., Morris, M., \& Cowley, S. 2005, ApJ, 631, 55

Bykov, A. M. 2002, A\&A, 390, 327

Coil, A. L., \& Ho, P. T. P. 2000, ApJ, 533, 259

Desert, F. X., Boulanger, F., Leger, A., Puget, J. L., \& Sellgren, K. 1986, A\&A, 159,328

Dwek, E., \& Smith, R. K. 1996, ApJ, 459, 686
Feigelson, E. D., \& Nelson, P. I. 1985, ApJ, 293, 192

García-Burillo, S., Martín-Pintado, J., Fuente, A., \& Neri, R. 2000, A\&A, 355, 499

Gray, A. D. 1994, MNRAS, 270, 861

Güsten, R., \& Philipp, S. D. 2004, in Proc. 4th Cologne-Bonn-Zermatt Symp. 91, The Dense Interstellar Medium in Galaxies, ed. S. Pfalzner, C. Kramer, C. Straubmeier, \& A. Heithausen (Berlin: Springer), 253

Handa, T., Sakano, M., Naito, S., Hiramatsu, M., \& Tsuboi, M. 2006, ApJ, 636, 261

Hüttemeister, S., Dahmen, G., Mauersberger, R., Henkel, C., Wilson, T. L., \& Martín-Pintado, J. 1998, A\&A, 334, 646

Hüttemeister, S., Wilson, T. L., Bania, T. M., \& Martín-Pintado, J. 1993, A\&A, 280,255

Irvine, W. M., Goldsmith, P. F., \& Hjalmarson, А. 1987, in Proc. Symp. on Interstellar Processes 134, Interstellar Processes, ed. D. J. Hollenbach \& H. A. Thronson, Jr. (Dordrecht: Reidel), 561

Jones, A. P., Tielens, A. G. G. M., \& Hollenbach, D. J. 1996, ApJ, 469, 740

Koyama, K., Inui, T., Matsumoto, H., \& Tsuru, T. G. 2008, PASJ, 60, S201

Koyama, K., Maeda, Y., Sonobe, T., Takeshima, T., Tanaka, Y., \& Yamauchi, S. 1996, PASJ, 48, 249

Koyama, K., Senda, A., Murakami, H., \& Maeda, Y. 2003, Chin. J. Astron. Astrophys., 3, 297

Koyama, K., Takikawa, Y., Hyodo, Y., Inui, T., Nobukawa, M., Matsumoto, H., \& Tsuru, T. G. 2009, PASJ, 61, S255

Leep, S., \& Dalgarno, A. 1996, A\&A, 306, L21

Li, A., \& Draine, B. T. 2001, ApJ, 550, L213

Maeda, Y., et al. 2002, ApJ, 570, 671

Maloney, P. R., Hollenbach, D. J., \& Tielens, A. G. G. M. 1996, ApJ, 466, 561

Martín, S., Requena-Torres, M. A., Martín-Pintado, J., \& Mauersberger, R. 2008, ApJ, 678, 245

Martín-Pintado, J., Bachiller, R., \& Fuente, A. 1992, A\&A, 254, 315

Martín-Pintado, J., de Vicente, P., Fuente, A., \& Planesas, P. 1997, ApJ, 482, L45

Martín-Pintado, J., Rodríguez-Fernández, N.J., Fuente, A., \& Planesas, P. 2000, A\&A, 356, L5

Meijerink, R., \& Spaans, M. 2005, A\&A, 436, 397

Meijerink, R., Spaans, M., \& Israel, F. P. 2007, A\&A, 461, 793

Mezger, P. G., Duschl, W. J., \& Zylka, R. 1996, A\&A Rev., 7, 289

Mitchell, J. B. A., Rebrion-Rowe, C., LeGarrec, J. L., Taupier, G., Huby, N., \& Wulff, M. 2002, A\&A, 386, 743

Morris, M., \& Serabyn, E. 1996, ARA\&A, 34, 645

Muno, M. P., Baganoff, F. K., Brandt, W. N., Park, S., \& Morris, M. R. 2007, ApJ, 656, L69

Muno, M. P., et al. 2003, ApJ, 589, 225

Murakami, H., Koyama, K., Sakano, M., \& Tsujimoto, M. 2000, ApJ, 534, 283

Murakami, H., Koyama, K., \& Tsujimoto, M. 2001, ApJ, 550, 297

Murakami, H., Senda, A., Maeda, Y., \& Koyama, K. 2003, Astron. Nachr., 324, 125

Predehl, P., Constantini, E., Hasinger, G., \& Tanaka, Y. 2003, Astron. Nachr., 324, 73

Park, S., et al. 2004, ApJ, 603, 548

Rodríguez-Fernández, N. J., Combes, F., Martín-Pintado, J., Wilson, T. L., \& Apponi, A. 2006, A\&A, 455, 963

Rodríguez-Fernández, N. J., Martín-Pintado, J., Fuente, A., de Vicente, P., Wilson, T. L., \& Hüttemeister, S. 2001, A\&A, 365, 174

Requena-Torres, M. A., Martín-Pintado, J., Rodríguez-Franco, A., Martín, S., Rodríguez-Fernández, N. J., \& de Vicente, P. 2006, A\&A, 455, 971

Serabyn, E., \& Güsten, R. 1987, A\&A, 184, 133

Siebenmorgen, R., Krugel, E., \& Mathis, J. S. 1992, A\&A, 266, 501

Spaans, M., \& Meijerink, R. 2005, Ap\&SS, 295, 239

Stäuber, P., Doty, S. D., van Dishoeck, E. F., \& Benz, A. O. 2005, A\&A, 440, 949

Sunyaev, R., \& Churazov, E. 1998, MNRAS, 297, 1279

Tsuboi, M., Handa, T., \& Ukita, N. 1999, ApJS, 120, 1

Tsuboi, M., Ukita, N., \& Handa, T. 1997, ApJ, 481, 263

Valinia, A., Tatischeff, V., Arnaud, K., Ebisawa, K., \& Ramaty, R. 2000, ApJ, 543,733

Voit, G. M. 1991, ApJ, 379, 122

Wilson, T. L., \& Rood, R. T. 1994, ARA\&A, 32, 191

Yan, M. 1997, PhD thesis, Harvard University

Yusef-Zadeh, F., Law, C., \& Wardle, M. 2002, ApJ, 568, L121

Yusef-Zadeh, F., Muno, M., Wardle, M., \& Lis, D. C. 2007, ApJ, 656, 847 